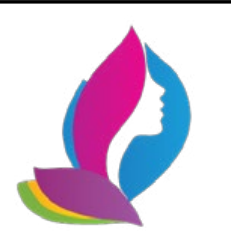

Contents List available at RAZI Publishing

Journal CleanWAS

Journal Homepage: http://www.razipublishing.com/journals/journal-cleanwas/

https://doi.org/10.26480/jcleanwas.01.2017.17.22

\title{
CURRENT ANALYTICAL METHODS FOR AMLODIPINE AND ITS FORMULATIONS: A REVIEW
}

A.B. M. Helal Uddina*, Mohamed Alaamaa, Zaidul I.S.M.a, S. A. Abbasb, Mohamed Awang.c, Fahim T.K. a

a Analytical and Bioanalytical Research Laboratory, Department of Pharmaceutical Chemistry, Faculty of Pharmacy, International Islamic

University Malaysia (IIUM), Jalan Istana, Bandar Indera Mahkota, 25200 Kuantan, Pahang, Malaysia.

b Faculty of Pharmacy, Quest International University Perak, Jalan Raja Permaisuri Bainun 30250 Ipoh, Perak Darul Ridzuan, Malaysia.

cFaculty of Pharmacy, Cyberjaya University College of Medical Sciences, 63000 Cyberjaya, Selangor DE, Malaysia. Corresponding author: abmhelal@iium.edu.my

This is an open access article distributed under the Creative Commons Attribution License, which permits unrestricted use, distribution, and reproduction in any medium, provided the original work is properly cited

\section{ARTICLE DETAILS}

Article history:

Received 27 September 2016

Accepted 13 December 2016

Available online 10 January 2017

Keywords:

Amlodipine, Antihypertensive, Analytical Method, Pharmaceuticals

\section{ABSTRACT}

The use of amlodipine is very common due to the effects of amlodipine on hypertension and coronary artery disease (CAD) like chronic stable angina, vasospastic angina (Prinzmetal's or variant angina), and angiographically documented CAD. Amlodipine is involved in several combinations with other antihypertensive drugs. The analysis of amlodipine and its co-drugs is reported using several analytical methods such as spectrophotometric, capillary electrophoresis and chromatographic methods. To our knowledge, there is no comprehensive reports which address all analytical methods for the analysis of amlodipine and combination, therefore we tried to gather as much as reports in one review paper to help researchers and industrial experts to easily access the information related to amlodipine analysis.

\section{INTRODUCTION}

Amlodipine is a third generation dihydropyridine calcium antagonist, It is used for the treatment of high blood pressure and angina [1]. It was firstly formulated by Pfizer under the name of Norvasc, and then several generic versions are available now. Amlodipine was combined with several drugs to enhance their activity.

2.0 Material \& Methods

\section{$2.1 \quad$ Chemical characteristics}

Amlodipine in pharmaceutical industry is found as besylate or maleate salts, and the most used is amlodipine besylate. Amlodipine besylate is a white crystalline powder; and its solubility in water and propanol is very poor, while it is freely soluble in methanol and sparingly soluble in ethanol. The melting range for amlodipine besylate is between $195-204^{\circ} \mathrm{C}$, while the melting range for amlodipine is $178-179^{\circ} \mathrm{C}[1]$.

\subsection{Pharmacokinetics}

The bioavailability of amlodipine when given orally is approximately $60 \%$ as it is well absorbed by the oral route. It is metabolized in the body largely to inactive pyridine compounds and about $60 \%$ of administered dose is excreted with the urine through renal elimination. The major metabolite identified in the urine was 2-([4-(2-chlorophenyl)-3-ethoxycarbonyl5-methoxycarbonyl-6-methyl- 2-pyridyl] methoxy) acetic acid, which represented $33 \%$ of amlodipine impurities secreted in the urine. The mean half-life of amlodipine in the plasma is $33 \mathrm{~h}$, while more time is required to eliminate of total drug-related material from plasma [2].
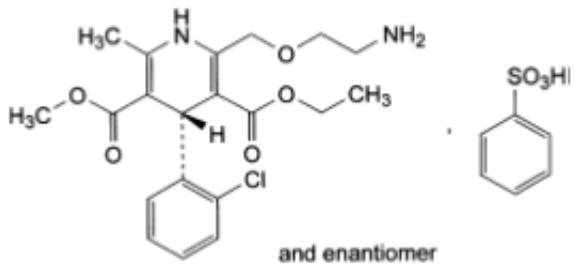

3-Ethyl 5-methyl (4RS)-2-[(2-aminoethoxy) methyl]-4-(2-chlorophenyl)-6methyl-1,4-dihydropyridine-3,5-dicarboxylate benzenesulphonate.

\subsection{Analytical methods for the analysis of amlodipine}

2.4.1 Spectrophotometric Methods for amlodipine analysis in different matrices

Derayea and co-authors developed and validated a new spectrophotometric method for the analysis of amlodipine and nicardipine in their raw materials and pharmaceutical formulation. Both drugs were reacted with eosin Y to form binary complex which show maximum absorptivity at $549 \mathrm{~nm}$. All parameters of the reaction such as temperature, $\mathrm{pH}$, and the surfactant were studied and optimized. Both drugs were dissolved in ethanol and distilled water to prepare the standard solutions, and the reaction was completed by mixing one milliliter of standard with $0.5 \mathrm{ml}$ of methyl cellulose surfactant $(0.3 \%)$ and $0.5 \mathrm{ml}$ of Mcllvaine buffer, one millilitre of eosin Y was added and the mixture was kept for 10 minutes in room temperature. After that the volume was brought to $10 \mathrm{ml}$ in volumetric flask. The analysis was performed using UV/Vis spectrophotometer at $549 \mathrm{~nm}$ wavelength. The results showed that the method was linear over the rang $5-60 \mu \mathrm{g} / \mathrm{ml}$ for amlodipine and $10-60 \mu \mathrm{g} / \mathrm{ml}$ for nicardipine. The limit of detection was 1.8 $\mu \mathrm{g} / \mathrm{ml}$ and $1.1 \mu \mathrm{g} / \mathrm{ml}$ for amlodipine and nicardipine respectively. LOQ was 6 $\mu \mathrm{g} / \mathrm{ml}$ and $5 \mu \mathrm{g} / \mathrm{ml}$ for amlodipine and nicardipine respectively. The method was checked for precision, accuracy and specificity and the results were satisfactory. The method was applied for the commercial formulation and compared with references methods. The recovery was 97.1- 99.8 and there is no significant difference between this method and reference methods. In conclusion authors suggested their method to be used in quality control laboratory because it is economic as it does no need expensive instruments or reagents. They claimed that their method is simple and it does no need for extraction. Overall the reported method is very sensitive and it can detect very low concentrations of these drugs [3].

Ragno and co-workers used third order derivative spectrophotometric methods for the analysis of amlodipine and its pyridine photodegradation product (AMLOX) using ethanol $95 \%$ as solvent at two wavelengths. They found the spectra of amlodipine and AMLOX are overlapping closely therefore they tested first to fourth derivative and found that third derivative is the best and gave less overlapping. Amlodipine was analysed at $243 \mathrm{~nm}$ without interference of its pyridine derivative. Amlodipine and AMLOX were analysed over the ranges $5 \mu \mathrm{g} / \mathrm{ml}-50 \mu \mathrm{g} / \mathrm{ml}$ and $0.2 \mu \mathrm{g} / \mathrm{ml}-5 \mu \mathrm{g} /$ $\mathrm{ml}$ respectively. The achieved LOD for AMLOX was $0.15 \mu \mathrm{g} / \mathrm{ml}$ while LOQ was $0.45 \mu \mathrm{g} / \mathrm{ml}$. This method can be applied immediately for dissolved and diluted tablet without any other treatment because the derivative spectra are not affected by background absorption resulted of turbidity baseline. They concluded that this method is simple and it can be applied to quality control for amlodipine pharmaceutical preparations [4].

Murat Uzturk and team developed spectrophotometric method to determine amlodipine besylate in the plasma without derivatization. Liquid - liquid extraction method was applied using mixture of ether-hexane 1-4 $\mathrm{v} / \mathrm{v}$ in buffer solution. Ethanol - acetonitrile $30-70 \%$ solution was used to prepare the sample and standards and the wavelength was 
adjusted to $360 \mathrm{~nm}$. The result revealed that the linearity range was between $2-17 \mu \mathrm{g} / \mathrm{ml}$ with LOD and LOQ of $1.5 \mu \mathrm{g} / \mathrm{ml}$ and $2 \mu \mathrm{g} / \mathrm{ml}$ respectively. Using this method of extraction, the recovery was $88.0 \%-98.4 \%$. The authors concluded that their method is rapid, simple, and accurate and it can be applied without any interference from the excipients [5].

Shyni Bernard and team developed a spectrophotometric method to estimate amlodipine besylate in pharmaceutical doses and bulk. They claimed that they solved the problem of using organic solvent by using $2 \mathrm{M}$ urea solution as hydrotropic solubilizing, as amlodipine is poorly soluble in water. The urea did not interfere the measurement in the working wavelength which was $243 \mathrm{~nm}$. The result showed that the LOD and LOQ were $2 \mu \mathrm{g} / \mathrm{ml}$ and 5 $\mu \mathrm{g} / \mathrm{ml}$ respectively in the linear range of $5-25 \mu \mathrm{g} / \mathrm{ml}$. The developed method was used for the analysis of amlodipine in two commercial formulations and the results showed good agreement between the tested and claimed amounts of amlodipine. The author concluded that this method is simple, precise, rapid and economic and it can be used for routine analysis of amlodipine in pharmaceutical formulations and biological fluids [6].

Patil and co-workers developed and validated two spectrophotometric methods for the determination of amlodipine besylate and losartan potassium in combined tablet dosage form. Methanol was used to dissolve two drugs and the spectra for two drugs showed that the $\lambda$ max were $208 \mathrm{~nm}$ and $237.5 \mathrm{~nm}$ for amlodipine besylate and losartan potassium respectively and the spectra also showed one iso absorptive point at $242.5 \mathrm{~nm}$. According to these spectra results, the first developed method was simultaneous equation method. In this method, two analytical wavelengths for both drugs which are $208 \mathrm{~nm}$ and $237.5 \mathrm{~nm}$ were used for the formation and solving a simultaneous equation. The second method was Absorbance ratio or Q-analysis method which was performed by measuring the absorptivity at $242.5 \mathrm{~nm}$ (as an iso-absorptive point) and $237.5 \mathrm{~nm}$. Two equations were prepared one for each method to calculate the concentrations of each drug The methods were validated and the linearity range was between $2-20 \mu \mathrm{g} /$ $\mathrm{ml}$ for both drugs. Methods were also applied for commercial tablets, and they gave agreement between calculated and proposed values for two drugs in commercial tablets with recovery of $95-110 \%$. The author concluded that these two methods are novel methods, rapid, simple, non-requiring extra extraction or heating, no organic solvents were used, and reproducible They can be applied for the routine analysis of Amlodipine besylate and losartan potassium in quality control analysis [7].

Two spectrophotometric methods were developed for another combination of amlodipine by Mishra and team. Amlodipine besylate and nebivolol hydrochloride were estimated in tablet form by these two methods. The drugs were prepared in methanol solution and scanned, and the spectra of two drugs showed maximum wavelengths of $238 \mathrm{~nm}$ and $360 \mathrm{~nm}$ for amlodipine and $281 \mathrm{~nm}$ for nebivolol. Results also showed no interference between two drugs in those wavelengths so for the first method $238 \mathrm{~nm}$ and $281 \mathrm{~nm}$ were used for amlodipine and nebivolol respectively in first method, while 360 and $281 \mathrm{~nm}$ were used for amlodipine and nebivolol respectively in the second method. The concentration of amlodipine was calculated immediately from the absorbance in both methods while the concentration of nebivolol was calculated using derived equations. The methods were statistically validated and tested for different range of standard concentrations. These two methods were applied for commercial tablets and the recovery study confirmed the reproducibility and reliability of these methods. The author concluded that these two methods are simple, accurate, sensitive and precise. Hence, they can be applied successfully in simultaneous determination of both drugs marketed formulations. Author also concluded that the first method was more accurate than the second one [8].

Bilal and co-workers developed a new spectrophotometric method to estimate amlodipine concentration using aromatic substitution reaction, as 2,4-Dinitrofluorobenzene (DNFB) react through a nucleophilic aromatic substitution reaction with amine group in amlodipine to give yellow product which have absorbance in $\lambda \max$ of $357 \mathrm{~nm}$. The reaction occurs in methanol solution in the existence of borate buffer to adjust $\mathrm{pH}$ to 7.8 with heating. The effect of $\mathrm{pH}$, the volume of DNFB, heating temperature and reaction time were studied and optimized. The method was validated according to USP 27 criteria. The linear rang used in this study was between $8-28 \mu \mathrm{g} / \mathrm{ml}$. LOD and LOQ were 0.58 and 1.93 respectively. The method was applied for commercial preparation and the recovery was much closer to what was claimed by the manufacturer and furthermore the method was compared with reference methods and the results were almost the same. This method was also used for other drugs in the same study which are acetylcysteine, captopril, and heptaminol hydrochloride. The reaction occurred on the amine group for heptaminol hydrochloride and amlodipine, while it occurred on the thiol group for acetylcysteine and captopril. The author concluded that this method is simple, as the reaction occurs directly to amine group in amlodipine without the need of prior conversion to the base or subsequent extraction. Moreover, there is no interference from the excipients or co drugs in combinations. Taking all these points in the mind and considering that some labs do not have modern instruments, the author recommend this method to be used in for routine quantitative determination of the amlodipine and other studied drugs in quality control laboratories [9].

Prasad and co-workers found new method for simultaneous determination of amlodipine and atenolol in their combination tablet using derivative spectroscopy. The drugs were dissolved in methanol and scanned for the absorbance in the range of wavelength $220-300 \mathrm{~nm}$. The first derivative spectra of the mixture showed zero cross point of 250 and $273.4 \mathrm{~nm}$ for amlodipine and 239 for atenolol where the absorbance of the solution is equal to the absorbance of one drug without any interfere from the other one. The study of any interference between drugs in the zero cross points was also reported by measuring the absorbance of pure and mixture drugs in these wavelengths. The wavelength used for amlodipine in this study was $250 \mathrm{~nm}$ and the absorbance was linear against the concentration in the range $2.5-10 \mu \mathrm{g} / \mathrm{ml}$, while LOD and LOQ were $0.020 \mu \mathrm{g} / \mathrm{ml}$ and $0.10 \mu \mathrm{g} /$ $\mathrm{ml}$ respectively. The method was applied for commercial tablets containing amlodipine and the results were very close to what was indicated by the manufacturers. The author concluded that derivative methods can help to solve the problem of estimating drugs in combined forms using spectrophotometry if there is overlapping spectra between them. Author also suggested this method to be used in quality control analysis labs as it is simple, rapid, accurate and reproducible. It is very important to mention that author used derivative spectroscopy to estimate haloperidol-tri hexy phenidyl in combined tablet preparations also in the same work [10].

New spectrophotometric and spectrofluorimetric method were developed and validated by Ayad and his co-workers for the simultaneous analysis of amlodipine besylate and doxazosin mesilate. The methods depend on the reaction of amlodipine besylate and doxazosin mesilate with acetyl acetone and formaldehyde to form a yellow compound which can be quantified either spectrophotometrically or spectrofluorimetrically. Standard and samples were prepared by dissolving amlodipine besylate and doxazosin mesilate in least amount of methanol and dilute with water. The reaction conditions were studied and optimized to give the optimum compound. All the parameters for the reaction such as $\mathrm{pH}$, heating time and the concentration of acetyl acetone and formaldehyde were studied and optimized. The methods were applied successfully on the commercial pharmaceutical form.

The spectrophotometric method was linear over the range of $6-44 \mu \mathrm{g} /$ $\mathrm{mL}$ for amlodipine besylate and $8-36 \mu \mathrm{g} / \mathrm{mL}$ for doxazosin mesilate. The linear range for spectrophotometric method was $1.6-7.6 \mu \mathrm{g} / \mathrm{mL}$ and $0.02-$ $0.22 \mu \mathrm{g} / \mathrm{mL}$ for amlodipine besylate and doxazosin methylate, respectively. The methods were studied for precision and accuracy and the results were in acceptable ranges. In conclusion, authors claimed that their method is simple, sensitive, precise and accurate. These methods can be used in routine analysis for studied drugs [11]

Nafisur Rahman and Nasrul Hoda developed and validated two spectrophotometric methods for the determination of amlodipine besylate in drug formulations. In first method, Amlodipine was reacted with 2 , 3-dichloro 5, 6 -dicyano 1, 4-benzoquinone (DDQ) to form coloured product which have maximum absorbance at $580 \mathrm{~nm}$. The second method depends on the reaction of drug with ascorbic acid in $\mathrm{N}, \mathrm{N}$-dimethylformamide medium (DMF) to produce coloured complex which have maximum absorbance at $530 \mathrm{~nm}$. Amlodipine besylate which is amino salt cannot react with DDQ immediately that is why amlodipine base (which can react with DDQ) was obtained by dissolving amlodipine besylate in chloroform and shacked with $0.5 \mathrm{M}$ aqueous sodium carbonate solution. The organic layer which contain amlodipine base was extracted and dried and dissolved in acetonitrile and reacted with DDQ. The produced complex was checked using IR. The concentration and the volume of DDQ were optimized. The coloured complex was found to be stable for 3-20 min. The results showed that the method was linear over the range of $1-125 \mu \mathrm{g} / \mathrm{ml}$, while the statistical analysis confirmed the accuracy and precision of this method. The method was applied to demonstrate amlodipine besylate concentration in known formulation and the results were compared with the standard methods and confirmed the accuracy and precision of the method.

The second complex was produced by the reaction of amlodipine besylate and ascorbic acid in DMF under high temperature. This complex was purple red in color and has maximum absorbance at $530 \mathrm{~nm}$. The effects of heating time and volume and concentration of ascorbic acid were studied and optimized. The linear range in this study was between $10-140 \mu \mathrm{g} /$ $\mathrm{ml}$. This method was also studied statistically and applied for commercial formulation sand compared with reference methods. The author concluded 
that the DDQ method is more precise and accurate than ascorbic acid method. Authors also recommended these two methods to be used in the analysis of amlodipine besylate in pharmaceutical dosage form because they are simple, sensitive, and reproducible [12].

Basavaiah and team developed indirect spectrophotometric method to determine amlodipine besylate in pharmaceuticals. This method depends on brominating amlodipine using fixed excess amount of bromated bromide mixture in acidic medium of $\mathrm{HCl}$. The remaining amount of bromine was reacted with fixed known amount of metanil yellow die and the remaining dye was determined by absorbance measuring at $530 \mathrm{~nm}$. All factors influencing the method were studied and validated including the concentration of die and amount of $\mathrm{HCl}$ and the time of taking the absorbance. The absorbance against the concentration of amlodipine was linear in the range of 1.25-7.20 $\mu \mathrm{g} / \mathrm{ml}$, while the LOD and LOQ were 0.17 and $0.56 \mu \mathrm{g} / \mathrm{ml}$ respectively. This method was applied for determination of amlodipine in two commercial formulations and there was close agreement between this method and reference methods. Amlodipine was determined in the same paper using HPLC method. The author concluded that those methods are simple and rapid. They also concluded that their spectrophotometric method did not need any heating or extracting [13].

Bahrami and Merzaeei developed and validated HPLC method with fluorescence detector for analysis of amlodipine in human serum. The amlodipine samples were introduced to C18 reversed phase column after derivatization with 4-chloro-7-nitrobenzofurazan. The internal standard used in this study was propranolol. Amlodipine samples were prepared using one step extraction by extracting amlodipine from serum by ethyl acetate. The samples were eluted using mobile phase of a mixture of sodium phosphate buffer $\mathrm{pH}$ (2.5) containing triethyl amine as a solvent $\mathrm{A}$ and methanol as a solvent $B$ in a flow rate of $2.8 \mathrm{ml} / \mathrm{min}$. The linear range was $0.25-16 \mathrm{ng} / \mathrm{ml}$. Standard stock solution was prepared by dissolving amlodipine in methanol. The method was applied for bioequivalence study as Amlodipine tablet of market leader and a generic equivalent were administered orally to 12 healthy volunteers in randomized single-dose cross-over study. Author concluded that this method is simple, fast, with higher resolution. The one step extraction procedure makes this method preferable compared to multiple extraction and washing steps in other previously published method [14].

Another study of spectrophotometric method for the determination of amlodipine besylate (ADB) in pure form and in pharmaceutical preparations was developed by Alizadeh and Hemati (2014). The methods are based on the charge transfer reactions between the drug as electron donor with 7,7,8,8-tetracyanoquinodimethane (TCNQ) and tetracyanoethylene (TCNE) as $\pi$-acceptors to the formation of charge transfer (CT) complexes. These reactions give coloured products which have maximum absorption bands at 745 and $396 \mathrm{~nm}$ for TCNQ and TCNE, respectively. Beer's law is obeyed in the concentration ranges $20-110 \mu \mathrm{g} \mathrm{mL}-1$ and 5-35 $\mu \mathrm{g} \mathrm{mL}-1$ for ADB using TCNQ and TCNE reagents. The molar absorptivities are $2.73 \times 103$ and $6.43 \times 103 \mathrm{~L} \mathrm{~mol}-1 \mathrm{~cm}$ and the Sandell (S) sensitivities are $0.14 \mu \mathrm{g} \mathrm{cm}-2$ and $0.063 \mu \mathrm{g} \mathrm{cm}-2$ using TCNQ and TCNE reagents, respectively, which indicate the high sensitivity of the proposed methods. The relative standard deviations (R.S.D.: 0.94 and 0.73) obtained using TCNQ and TCNE reagents, respectively, refer to the high accuracy and precision of the proposed method [15].

Kadioglu and Ozturk (2012) developed a rapid and sensitive spectrofluorimetric method for the determination of amlodipine (AD) in the plasma. The calibration curves were linear $(\mathrm{r} 2 \geq 0.997)$ in the concentration range of 0.1-12.5 ppm of AD. The limit of quantitation and limit of detection values for the method for plasma samples were $0.1 \mathrm{ppm}$ and $0.07 \mathrm{ppm}$, respectively. The precision calculated as the relative standard deviation was less than $3.5 \%$, and the accuracy (relative error) was better than $5.5 \%$ $(\mathrm{n}=6)$. The method developed in this study can be directly and easily applied for the determination of $\mathrm{AD}$ in the plasma without derivatization in plasma [16].

Another study of kinetic spectrophotometric method developed and validated by Mahmoud et al. (2012) to determine amlodipine besylate in its pharmaceutical tablets. The method was based on the condensation reaction of AML with 7-chloro-4-nitro-2,1,3-benzoxadiazole in an alkaline buffer ( $\mathrm{pH}$ 8.6) producing a highly-colored product. The color development was monitored spectrophometricaly at the maximum absorption $\lambda \max 470$ $\mathrm{nm}$. Here, both the activation energy and the specific rate constant $\left(\right.$ at $\left.70^{\circ} \mathrm{C}\right)$ of the reaction were found to be $6.74 \mathrm{kcal}$ mole- 1 and $3.58 \mathrm{~s}-1$, respectively. Under the optimum reaction conditions, the limits of detection and quantification were 0.35 and $1.05 \mu \mathrm{g} / \mathrm{mL}$, respectively. The precision of the method was satisfactory; the relative standard deviations were $0.85-1.76 \%$. The proposed method was successfully applied to the analysis of AML in its pure form and tablets with good accuracy; the recovery percentages ranged from $99.55 \pm 1.69 \%$ to $100.65 \pm 1.48 \%$. The results were compared with that of the reported method [17].

\subsubsection{Chromatographic Methods for amlodipine in different matrices} TLC methods for amlodipine in different matrices

Argekar and Powar published and validated new high-performance thinlayer chromatography HPTLC method for the simultaneous analysis of atenolol and amlodipine in tablet dosage form. In this method, the mobile phase consisted of a solution of methylene chloride: methanol: ammonia solution $(25 \% \mathrm{NH})$ (8.8:1.3:0.1; v: v). The stationary phase which used for separation was Merck HPTLC plates $(0.2 \mathrm{~mm}$ thickness) pre-coated with 60 F254 silica gel on aluminium sheet. The detection was performed using UV Densitometric detector at $230 \mathrm{~nm}$. The result showed linearity between amlodipine concentrations and the plot of peak areas in the range of $10-500 \mu \mathrm{g} / \mathrm{ml}$. The LOD and LOQ for amlodipine were 0.2 and $0.6 \mu \mathrm{g} /$ $\mathrm{ml}$ respectively. This method was applied for two commercial dosage forms and the results were close to what were claimed by the manufacturer, furthermore commercial tablets were spiked with known amount of amlodipine standard and the recovery was more than 98\%, confirming the accuracy and precision of this method. The authors concluded that this method is simple easy to perform, accurate and precise. More samples can be analysed in the same time and this method can be extended to other pharmaceutical preparations for these two drugs [18].

Dhaneshwar and co-workers developed TLC method for the determination of amlodipine besylate and valsartan simultaneously in bulk drug and dosage form. Drug standards and sample solutions were prepared in methanol and spotted to aluminium plates pre-coated with silica gel 60 F254. A combination of toluene: methanol: acetic acid 7:3:0.1 (v/v/v) was used as mobile phase in this study. The densitometric measurement of the intact spot was performed at $244 \mathrm{~nm}$ for the detection. The linear range for this method was between 100-600 ng/spot, and LOD and LOQ were 50 and $100 \mathrm{ng} / \mathrm{spot}$ respectively. According to the authors, the validated method was successfully applied for the determination of the drug in bulk powder, and a commercial sample. They suggested their method to be used in studying the degradation of amlodipine and furthermore to estimate amlodipine concentration in plasma and biological fluids [19].

Meyyanathan and Suresh developed a high-performance thin-layer chromatographic (HPTLC) method for simultaneous quantitative analysis of amlodipine and benazepril in pharmaceutical formulations using zolpidem as an internal standard. Separation was performed on aluminium HPTLC plates coated with a layer of silica gel 60 F254 and prewashed with methanol. The mobile phase used in this method consisted of methanol-toluenetriethylamine 1:3.5:0.1 (v/v). Densitometric detection and quantification was performed at $254 \mathrm{~nm}$. Results achieved showed linear range of 0.1-0.8 $\mu \mathrm{g} / \mathrm{spot}$, while LOD and LOQ were 0.02 and $0.08 \mu \mathrm{g} / \mathrm{spot}$ respectively. The authors concluded that the method can be applied for routine quality-control analysis of amlodipine and benazepril in pharmaceutical preparations, because it is accurate, precise, rapid, and selective [20].

Patel and co-workers developed and validated new HPTLC method for the simultaneous analysis of amlodipine besylate and indapamide in pharmaceutical dosage form. Plates precoated with silica gel 60 F245 were used as stationary phase and they were developed by mobile phase which consisted of methanol-ethyl acetate - toluene-ammonia 2.5:3.5:4:0.2\% $/ \mathrm{v} / \mathrm{v}$. Densitometric detection was performed at $343 \mathrm{~nm}$ for the determination of both drugs. The results revealed that the method was linear over the range 250-2500 ng/spot for amlodipine and 150-1500 ng/spot for indapamide. Standard solutions and samples were prepared using methanol as a solvent. The method was validated per ICH criteria, and the LOD was $76.78 \mathrm{ng} /$ spot and $30.09 \mathrm{ng} / \mathrm{spot}$ for amlodipine and indapamide respectively, while the LOQ was $230.36 \mathrm{ng} / \mathrm{spot}$ and $92.08 \mathrm{ng} / \mathrm{spot}$ for amlodipine and indapamide respectively. the method was evaluated for precision, accuracy and repeatability and the results were in the accepted ranges. The method was applied for commercial pharmaceutical formulation and the results were satisfactory. The authors concluded that this method is simple rapid and economic due to the possibility of running large number of samples in the same time [21].

HPLC and UPLC methods for amlodipine in different matrices

RP-HPLC method was developed by Yeung to determine amlodipine in plasma in the presence of desipramine hydrochloride as internal standard. Liquid extraction was used to prepare samples by extracting amlodipine using methyl tert-butyl ether and the samples were injected to C18 reversed phase column (250X4.1mm id). Samples were eluted using a mixture of methanol, ammonium acetate-acetonitrile (38:38:24, v/v/v) containing $0.02 \%$ triethylamine, and the glacial acetic acid was used to adjust $\mathrm{pH}$ to 7.1 , and the flow rate was $1.2 \mathrm{ml} / \mathrm{min}$. Samples were determined using UV 
detector at the wavelength of $240 \mathrm{~nm}$. Under these conditions, the results showed the retention times of amlodipine and the internal standard desipramine were 10.6 and 12.19 min respectively. The Method was linear over the range of 2.5 to $100 \mathrm{ng} / \mathrm{ml}(\mathrm{R} 2=0.990)$. [22].

Massaroti and his team developed and validated new LC-MS-MS method for the determination of amlodipine in human plasma using nimodipine as internal standard. Samples were extracted from the plasma using liquid extraction and injected to C18 (50 X $2 \mathrm{~mm})$ column. A solution of $0.1 \%$ formic acid in acetonitrile: water $(80-20 \% \mathrm{v} / \mathrm{v})$ was used as mobile phase and the flow rate was $0.15 \mathrm{ml} / \mathrm{min}$. For detection, Mass spectrometric detector was used using Quattro Micro equipment working with an ESI source in the positive ion mode. The standard of amlodipine and nimodipine were prepared using acetonitrile. All extraction and separation conditions were optimized. The results showed that linear range of the method was between $0.2-20 \mathrm{ng} / \mathrm{ml}$. the method was very raped as retention time was 1.9 for amlodipine and $3 \mathrm{~min}$ for nimodipine. The method was validated and all the results were satisfactory[23].

Shimooka and co-authors developed RP-HPLC method to determine amlodipine besylate in human serum using amperometric detector. N,ZVdimethyl analogue was used as internal standard (IS) and amlodipine standards were prepared by dissolving amlodipine besylate salt in distilled water. Samples were extracted from serum by liquid extraction using diethyl ether. Samples were injected to $\mathrm{C} 18$ reversed phase column and eluted using mobile phase of $0.05 \mathrm{M}$ phosphate buffer solution ( $\mathrm{pH}$ 3.1)-acetonitrile $(65: 35, v / v)$ containing sodium octane sulphonate and EDTA at final concentrations of $0.005 \mathrm{M}$ and $5 \mathrm{mg} / \mathrm{l}$ respectively. The flow rate was $1 \mathrm{ml} /$ min and the detector was amperometric detector. The method was linear over the range $0.2-2 \mathrm{ng} / \mathrm{ml}$. the method was applied successfully to study the pharmacokinetic of amlodipine in human plasma and the results were comparable with known methods. Authors concluded that this method can be used to analyse other dihydropyridine compounds such as nifedipine and nicardipine [24].

Chitlang and team developed and validated new RP-HPLC method for the simultaneous determination of amlodipine and valsartan in capsules formulation. The mobile phase consisted of acetonitrile: potassium dihydrogen phosphate buffer $(0.02 \mathrm{M}, \mathrm{pH} 3.0)(56: 44 \mathrm{v} / \mathrm{v})$ and the samples were injected into RP C-18 Column (Kromasil, $250 \times 4.6 \mathrm{~mm}$ ). the flow rate was $1 \mathrm{ml} / \mathrm{min}$ and the samples were analysed using UV detector and the wavelength was set to $234 \mathrm{~nm}$. standards and samples were prepared by dissolving both drugs in the mobile phase. Both drugs were analysed using this method after applying different types of stress for degradation studies. The method was linear over the range $1-40 \mu \mathrm{g} / \mathrm{ml}$ and $10-80 \mu \mathrm{g} / \mathrm{ml}$ for amlodipine and valsartan respectively. LOD was $0.03 \mu \mathrm{g} / \mathrm{ml}$ and $0.018 \mu \mathrm{g} / \mathrm{ml}$ while LOQ was $0.089 \mu \mathrm{g} / \mathrm{ml}$ and $0.054 \mu \mathrm{g} / \mathrm{ml}$ for amlodipine and valsartan respectively. The results of precision, accuracy and recovery studies were in the acceptable limits. In conclusion the method is simple, raped and precise and it can be used for the simultaneous analysis of amlodipine and valsartan in pharmaceutical formulations [25].

El-Gizawy and co-authors developed and validated HPLC method for the simultaneous analysis of Amlodipine besylate Valsartan and Hydrochlorothiazide in their combination and in spiked human plasma. Samples were prepared by dissolving drugs mobile phase which consist of acetonitrile - phosphate buffer (40-60) with pH 2.8. Samples were injected into $\mathrm{C} 18$ reversed phase column and the flow rate was $0.8 \mathrm{ml} / \mathrm{min}$. UV detector was used for the detection of the drugs at $227 \mathrm{~nm}$ wavelength Plasma samples were prepared by spiking $0.1 \mathrm{ml}$ of plasma by $100 \mathrm{ul}$ of standard solution and the volume was brought to $5 \mathrm{ml}$ using acetonitrile Plasma samples were centrifuged and further dilution was made by mobile phase. The results showed that the retention time was 2.26, 3.16 and 11.19 min for hydrochlorothiazide, amlodipine, and valsartan respectively. The linear range was $4-28 \mu \mathrm{g} / \mathrm{ml}, 5-40 \mu \mathrm{g} / \mathrm{ml}$ and $1-12 \mu \mathrm{g} / \mathrm{ml}$ for amlodipine, Valsartan and Hydrochlorothiazide respectively. The method was applied for commercial pharmaceutical formulation and the result was satisfactory without interference of excipients. The method was checked for precision, accuracy and specificity and the results were in the acceptable levels. [26]. Jossefen and co-workers developed a new HPLC method depending on a narrow bore HPLC assay with electrochemical detection for the determination of amlodipine in human plasma. The column used was a Zobrax SB-Phenyl column $150 \times 2.1 \mathrm{~mm}$. the chromatography was carried out using a mixture of methanol and $0.1 \mathrm{M}$ acetate buffer, $\mathrm{pH} 4$ in a ratio $65: 35(\mathrm{v} / \mathrm{v})$ as a mobile phase under a flow rate of $0.3 \mathrm{ml} / \mathrm{min}$. The internal standard which used in this study was UK52.829. The detection was carried out electrochemically with the applied voltage of 0.59 volt on the analytical cell, and nanoampere sensitivity was used. Amlodipine was extracted from plasma by solid phase extraction using bond elute C2 column and $2.5 \%$ ammonia in acetonitrile as a solvent. In this study, the elution time required for both amlodipine and internal standard was $15 \mathrm{~min}$. The method was linear over the range $0.5-20 \mathrm{ng} / \mathrm{ml}$ and LOD was $0.2 \mathrm{ng} / \mathrm{ml}$. The recovery of extraction method was reported to be $89 \%$ for amlodipine and $95 \%$ for internal standard [27].

Naidu and co-workers developed and validated new RP - HPLC method for simultaneous determination of amlodipine besylate and benazepril hydrochloride in their combined pharmaceutical dosage form. This method was also used mainly to determine the degradation in these two drugs in their samples. The degrades resulted from thermal, photolytic, hydrolytic and oxidative degradation were studied by this method. Chromatographic conditions used were optimized and chosen as the column used in this study was Zorbax SB C18, $5 \mathrm{um}, 250 \mathrm{~mm} \times 4.6 \mathrm{~mm}$ i.d. Phosphate buffer and acetonitrile in the proportion of $65: 35(\mathrm{v} / \mathrm{v})$ with $\mathrm{pH}$ adjusted to 7.0 was used as mobile phase, and the detector was photodiode array detector with wavelength of $240 \mathrm{~nm}$. Methanol and mobile phase were used to prepare the standard solutions and tablet samples. The results showed linearity for amlodipine over the range of $6-14 \mu \mathrm{g} / \mathrm{ml}$. The recovery study approved the accuracy of the method and statistically study showed the precision of this method. The method showed good resolution between two drugs and their degradants in all degradation methods. The authors concluded that this method is specific, accurate and precise and it can be used as stability indicating method for assay of amlodipine besylate and benazepril hydrochloride in their combination product [28].

Donger and co-workers developed and validated new reverse phase HPLC method for the simultaneous the analysis of metoprolol succinate (MS) and amlodipine besylate $(\mathrm{AB})$ in tablet dosage form. Chromatography was carried out using Hypersil BDS cyano $(250 \mathrm{~mm} \times 4.6 \mathrm{~mm}, 5 \mathrm{~m})$ column. The detection was achieved using PDA detector at $254 \mathrm{~nm}$. Two drugs were eluted using isocratic mobile phase consisting of a mixture of aqueous triethylamine buffer $\mathrm{pH} 3$ and acetonitrile in the ratio of 85:15 (v/v) and phosphoric acid was used to adjust the $\mathrm{pH}$. The flow rate was $1.0 \mathrm{~mL} / \mathrm{min}$. Standard solutions and sample solutions were prepared using methanol and mobile phase. The method was validated according to ICH guidelines and the method was found linear over the range $42-98 \mu \mathrm{g} / \mathrm{ml}$. The authors concluded that their method is specific, accurate, rapid and precise. Authors recommended their method to be used for routine analysis of amlodipine besylate and metoprolol succinate in quality control analysis of pharmaceutical preparations containing these drugs [29].

Ma and co-workers validated a UPLC-MS/MS method for the determination of amlodipine in human plasma and employed it in pharmacokinetic studies. Chromatography was performed on an Acquity UPLC BEH C18 column $(50 \mathrm{~mm} \times 2.1 \mathrm{~mm}$, i.d., $1.7 \mathrm{um})$ with a mobile phase of water and acetonitrile (each containing $0.3 \%$ formic acid) under gradient conditions and a flowrate of $0.35 \mathrm{ml} / \mathrm{min}$. The volume injected was $5 \mu \mathrm{L}$ and the detection was performed on a triple-quadruple tandem mass spectrometer by multiple reactions monitoring (MRM) mode via Turbo ion spray ionization (ESI). The internal standard used in this study was Nimodipine and all standards were prepared in methanol water $50: 50 \mathrm{~V} / \mathrm{V}$, while the plasma samples were prepared by extraction amlodipine and internal standard by diethyl ether and drying them, then dissolving them in acetonitrile. The method was validated and the linear range was found $0.15-16 \mathrm{ng} / \mathrm{ml}$, while the LOQ was $0.15 \mathrm{ng} / \mathrm{ml}$. The stability of amlodipine in stored and frozen samples was also studied. The method was applied for pharmacokinetic study of amlodipine. The amlodipine concentrations were determined in healthy volunteers' plasma who have taken two tablets of amlodipine containing $10 \mathrm{mg}$ before and 1.0, 2.0, 3.0, 4.0, 5.0, 6.0, 8.0, 10.0, 14.0, 24.0, 48.0, 72.0, 96.0 and $120 \mathrm{~h}$ post-dosing. The results of pharmacokinetic study agreed with what was described in the literature. According to the author the run time was $3 \mathrm{~min}$ and the result was sensitive, accurate, and selective for amlodipine determination in human plasma [30].

Kasawar and Farooqui developed and validated new UPLC method for simultaneous determination of amlodipine besylate and benazepril hydrochloride in pharmaceutical dosage form. Column used for separation was an Acquity UPLC, BEH C8 (100 mm x $2.1 \mathrm{~mm}, 1.7$ micron) column, and the detector was a photodiode array detector. The separation was achieved by using mixture of $0.01 \mathrm{M}$ phosphate buffer $\mathrm{pH} 3.0$ and mixture solvent of equal amount of acetonitrile and methanol in the ratio of 45:55 $(\mathrm{v} / \mathrm{v})$ as a mobile phase. The flow rate was $0.3 \mathrm{ml} / \mathrm{min}$. To prepare the samples, phosphate buffer, water and methanol were used. To optimize chromatographic conditions, several types of column and several mixtures of mobile phase were studied. The results showed good resolution between two drugs and the peaks were free of tailing. The linear range was 5.21$15.63 \mu \mathrm{g} / \mathrm{ml}$, and LOQ was $0.01 \mu \mathrm{g} / \mathrm{ml}$ for amlodipine besylate. The authors concluded that this method is accurate, specific, precise and sensitive for the determination of amlodipine and benazepril in their capsules dosage. This method is suitable for determination of both drugs in their pharmaceutical 
dosage as the method is comparably easy and economical as the solvent consumption is very low [31].

Barman and team developed and validated reversed phase high performance liquid chromatography for the determination of amlodipine and atenolol in pharmaceutical dosage forms. Chromatographic separation was carried out using C18 reversed phase column and the mobile phase consisted of ammonium acetate buffer, acetonitrile and methanol in a ratio of 35:30:35 $\mathrm{V} / \mathrm{V}$. The flow rate was $1.5 \mathrm{ml} / \mathrm{min}$ and the detection was achieved using UV detector at $237 \mathrm{~nm}$. Amlodipine and atenolol were eluted in the temperature of $400 \mathrm{C}$. The elution time was $3.4 \mathrm{~min}$ for amlodipine. The method was validated according to ICH guideline and it was applied for commercial tablets containing both drugs and the results were close to what was stated by the manufacturer. The author concluded that this method is rapid, precise and simple and it can be used in routine analysis for dosage forms containing these two drugs [32].

$\mathrm{Yu}$ and co-workers developed and validated high performance liquid chromatography - tandem mass spectrometry for the determination of amlodipine besylate and atorvastatin in hypertension patients' plasma. Chromatographic conditions were as follow: the column used was Eclipse XDB-C18 2.1 X $100 \mathrm{~mm}, 3.5 \mathrm{um}$ column. The mobile phase was a mixture of $0.1 \%$ of formic acid in water solvent $\mathrm{A}$ ) and $0.1 \%$ of formic acid in acetonitrile (solvent B) in gradient program. The flow rate was $0.4 \mathrm{ml} / \mathrm{min}$. the detector used in this study was tandem mass spectrometry with electrospray ionization (ESI) interface in positive ion mode. Nitrindipine was used as internal standard. All standard solutions were prepared using acetonitrile while spiked plasma samples were extracted using methanol precipitation. The matrix effect and the stability of the analytes were studied also. The complete run was $7 \mathrm{~min}$. The results revealed linear range of $0.46-1000 \mathrm{ng} /$ $\mathrm{ml}$ for amlodipine and LLOQ was $0.46 \mathrm{ng} / \mathrm{ml}$. the method was validated and the extraction recovery was determined to be $93.3-101.7 \%$. The method was applied to study amlodipine and atorvastatin concentration in patients' plasma after administration and the results were satisfactory. The authors concluded that this method is rapid, accurate, sensitive and consume small amount of samples $50 \mathrm{ul}$ of plasma [33].

Nirogi and team developed and validated liquid chromatography/tandem mass spectrometry assay for the analysis of amlodipine in human plasma. According to the author this method was developed to get very low LLOQ which is $50 \mathrm{pg} / \mathrm{ml}$ of amlodipine in human plasma. Samples were injected into a reverse phase $\mathrm{C} 18$ column. A mixture of water-acetonitrile-formic acid $(30: 70: 0.03, \mathrm{v} / \mathrm{v})$ was used as mobile phase with a flow rate of $0.1 \mathrm{ml} /$ $\mathrm{min}$. The detection was achieved using MS detection in the multiple reaction monitoring mode. Tamsulosin was used as internal standard. The standard solutions were prepared using methanol and diluted in methanol- water $50: 50 \mathrm{v} / \mathrm{v}$. The plasma samples were prepared by liquid-liquid extraction using diethyl ether- dichloromethane 7:3 v/v and drying the organic layer and then dissolving it in methanol-water $50: 50 \mathrm{v} / \mathrm{v}$. The stability study of amlodipine in human plasma and the extraction recovery were studied using this method. This method was applied in pharmacokinetic study for healthy volunteers. The results showed linear range of 50-10000 pg/ $\mathrm{ml}$ of amlodipine in human plasma and LLOQ achieved was $50 \mathrm{pg} / \mathrm{ml}$. The recovery of extraction was $74.7 \pm 4.6 \%$. The total time for the run was 1.5 min. The author concluded that this method is simple, rapid (run of 1.5 min), and economic [34].

Bhatt and co-workers developed and validated new LC-MS/MS method for the determination of amlodipine in human plasma. The method utilizes Hypersil BDS C18 column and mass spectrophotometry as a detector. Imepramin was used as internal standard. The mobile phase consisted of methanol and ammonium format ( $\mathrm{pH} 4.5,10.0 \mathrm{mM}$; 80:20) with a flow rate of $0.5 \mathrm{~mL} / \mathrm{min}$. standard solution were prepared using methanol as a solvent and diluted with water - methanol $70: 30 \mathrm{v} / \mathrm{v}$ to get work solutions. Solid phase extraction was used to prepare the samples from the plasma as amlodipine was eluted using $0.2 \%$ acetic acid in methanol. The total time for run was $3.2 \mathrm{~min}$ and the linear range was between $0.1-10 \mathrm{ng} / \mathrm{ml}$ with LOQ of $0.1 \mathrm{ng} / \mathrm{ml}$. the recovery and stability studies were done using this method. The authors concluded that this method is rapid sensitive accurate and simple, therefore it can be used in determination of amlodipine in human plasma for pharmacokinetic and bioequivalence studies [35]

A new LC-MS-MS method for the simultaneous determination of amlodipine, losartan and losartic acid in human plasma was developed and validated by Karra and co-workers. Irbisartan was used as internal standard and the separation nvb was conducted in a C18 column. The mobile phase consisted of a mixture of methanol and $0.1 \% \mathrm{v} / \mathrm{v}$ formic acid $85: 15 \% \mathrm{v} / \mathrm{v}$. The results were reported using mass spectrophotometer detector in positive ionization mode. Samples were prepared by extracting the analytes from human plasma by solid phase extraction. The results showed that $2.5 \mathrm{~min}$ is enough for the three analytes and internal standard to be eluted with good resolution. The linear range was $0.05-10.1 \mathrm{ng} / \mathrm{ml}$ for amlodipine and $0.5-1000 \mathrm{ng} / \mathrm{ml}$ for losartan and losartic acid. The method was validated according to FDA guidelines and all parameters were in the accepted limits. The method was applied for human plasma for kinetic studies and the results were satisfactory [36].

Amlodipine in human plasma was analysed by Feng teamwork using LC-MS. Necardipine was used as internal standard and both drugs were extracted from plasma using ethyl acetate. Samples were injected into C18 column and the mobile phase was methanol - $1 \%$ glacial acetic acid $(65-35 \% \mathrm{v} / \mathrm{v})$. The temperature which used for the analysis was set to $35^{\circ} \mathrm{C}$ and the flow rate was $0.8 \mathrm{ml} / \mathrm{min}$. For detection, an air pressure ionization single quadruple mass spectrometer equipped with an ESI interface was used and it was operated in positive-ionization mode. Methanol was used to prepare standard stock solutions. Samples were prepared and extracted by spiking $1 \mathrm{ml}$ of plasma with amlodipine and internal standard and extracting it by adding $\mathrm{NaOH}(1 \mathrm{M})$ and ethyl acetate. The organic layer was separated and evaporated and the remaining were dissolved in methanol to inject in the column. The results showed that the method was linear over the range of $0.1-20 \mathrm{ng} / \mathrm{ml}$. the results of precision, accuracy and recovery were satisfactory. The method was applied to analyse amlodipine in human plasma for $10 \mathrm{mg}$ dose study and the results were like those reported by previous methods. The authors concluded that their method is simple, fast, accurate and precise and the extraction method is simple. The use of MS detector gave the method more specificity and selectivity [37].

\subsection{Conclusion}

Due to its importance and intensive use in medical field, amlodipine received much attention in research and industry. There are number of generic formulations and combinations of amlodipine and other antihypertensive drugs available in the market. Analytical method for these combination drugs are not listed in the pharmacopeia yet. To optimize the analytical method, it becomes very difficult for the analyst to design and fulfil the specification of the drug. So far there are several reports on the analysis of amlodipine and its formulation following spectroscopic, chromatographic and capillary electrophoresis methods using wide range of detectors such as UV, diode array, fluorescence, electrochemical, and MS detectors. In this work, we strive to gather as many methods related to amlodipine analysis suitable for the researchers to extract important information to establish their own method. There are still lacks of proper analytical method which can comply with the international guideline for the analysis of the drugs. In coming days' researcher and industries are expected to come out with more suitable analytical methods for the analysis of amlodipine and its formulation. It is very important to mention that the door is still open for more work in analysis side of amlodipine either in research or industrial set up.

\section{Acknowledgement}

The authors expressed their gratitude to staff of IIUM Pharmaceutical Manufacturing Plant and Department of Pharmaceutical Chemistry, Faculty of Pharmacy, International Islamic University Malaysia for their help and support. This research was supported by several grants from Research Management Center, International Islamic University Malaysia (EDW B and RIGS), Ministry of Higher Education (MOHE) (RACE) and Ministry of Finance (MOF).

\section{References}

[1] The British Pharmacopoeia, V., Her Majesty's Stationery Office. 2008, London, UK.

[2] Beresford, A.P., et al., Metabolism and kinetics of amlodipine in man. Xenobiotica, 1988. 18(2): p. 245-254.

[3] Derayea, S.M., et al., Spectrophotometric determination of amlodipine and nicardipine in pharmaceutical formulations via binary complex formation with eosin Y. Journal of Applied Pharmaceutical Science, 2012. 2(06): p. 84-89.

[4] Ragno, G., A. Garofalo, and C. Vetuschi, Photodegradation monitoring of amlodipine by derivative spectrophotometry. Journal of pharmaceutical and biomedical analysis, 2002. 27(1-2): p. 19-24.

[5] Ozturk; M. and Y. Kadioglu., developement and validation of UV spectroscopy method for determine amlodipine besylate in the plasma without derivatization. J. Pharm. Sci., 2007. 6: p. 1-6.

[6] Bernard, S., M. Mathew, and K. Senthilkumar, Spectrophotometric method of estimation of Amlodipine besylate using hydrotropic solubilization. Journal of Applied Pharmaceutical Science, 2011. 1(09): p. 177-180.

[7] Patil, P.R., et al., Simultaneous UV Spectrophotometric Method for Estimation of Losartan Potssium and Amlodipine Besylate in Tablet Dosage 
Form. Asian J. Research Chem, 2009. 2(1): p. 183-187.

[8] Mishra, P., K. Shah, and A. Gupta, Spectrophotometric methods for simultaneous estimation of nebivolol hydrochloride and amlodipine besylate in tablets. International J Pharm Pharm Sci, 2009. 1(2): p. 55-61.

[9] Belal, S.F., R.S. Haggag, and R.A. Shaalan, The Use of an Aromatic Substitution Reaction in the Spectrophotometric Determination of Selected Amino or Thiol Containing Drugs. Journal of Food and Drug Analysis, 2008. 16(1): p. 26-33.

[10] Prasad, C., et al., Simultaneous Determination of Atenolol-Amlodipine and Haloperidol-Trihexyphenidyl in Combined Tablet Preparations by Derivative Spectroscopy. Pharmacy and Pharmacology Communications, 1998. 4(7): p. 325-330.

[11] Ayad, M., et al., Spectrophotometric and spectrofluorimetric determination of amlodipine besilate and doxazosin mesilate in bulk and in dosage forms via Hantzsch reaction. Int J Pharm, 2012. 3(2): p. 111-116. [12] Rahman, N. and M. Nasrul Hoda, Validated spectrophotometric methods for the determination of amlodipine besylate in drug formulations using 2,3-dichloro 5,6-dicyano 1,4-benzoquinone and ascorbic acid. Journal of pharmaceutical and biomedical analysis, 2003. 31(2): p. 381-392.

[13] Basavaiah, K., U. Chandrashekar, and P. Nagegowda, Spectrophotometric and high performance liquid chromatographic determination of amlodipine besylate in pharmaceuticals. Sci. Asia, 2005. 31: p. 13-21.

[14] Bahrami, G. and S. Mirzaeei, Simple and rapid HPLC method for determination of amlodipine in human serum with fluorescence detection and its use in pharmacokinetic studies. Journal of pharmaceutical and biomedical analysis, 2004. 36(1): p. 163-168.

[15] Alizadeh, N. and F. Hemati, Spectrophotometric method for the determination of amlodipine besylate in pure and dosage forms using 7,7 , 8, 8-tetracyanoquinodimethane and tetracyanoethylene. Bulletin of Faculty of Pharmacy, Cairo University, 2014. 52(1): p. 109-114.

[16] Kadioglu, Y. and M. Ozturk, Spectrofluorimetric determination of amlodipine in human plasma without derivatization. Brazilian Journal of Pharmaceutical Sciences, 2012. 48(4): p. 719-725.

[17] Mahmoud, A.M., H.M. Abdel-Wadood, and N.A. Mohamed, Kinetic spectrophotometric method for determination of amlodipine besylate in its pharmaceutical tablets. Journal of Pharmaceutical Analysis, 2012. 2(5): p. 334-341.

[18] Argekar, A. and S. Powar, Simultaneous determination of atenolol and amlodipine in tablets by high-performance thin-layer chromatography. Journal of pharmaceutical and biomedical analysis, 2000. 21(6): p. 1137 1142.

[19] Dhaneshwar, S.R., N.G. Patre, and M.V. Mahadik, Validated TLC Method for Simultaneous Quantitation of Amlodipine Besylate and Valsartan in Bulk Drug and Formulation. Chromatographia, 2009. 69(1): p. 157-161.

[20] Meyyanathan, S. and B. Suresh, HPTLC method for the simultaneous determination of amlodipine and benazepril in their formulations. Journal of chromatographic science, 2005. 43(2): p. 73-75.

[21] Patel, D.B., Simultaneous Estimation of Amlodipine Besylate and Indapamide in Pharmaceutical Formulation by Thin-Layer Chromatographic-Densitometric method. Novel Science International Journal of Pharmaceutical Sciences, 2012. 1(2).

[22] Yeung, P.K.F., S.J. Mosher, and P.T. Pollak, Liquid chromatography assay for amlodipine: Chemical stability and pharmacokinetics in rabbits. Journal of Pharmaceutical and Biomedical Analysis, 1991. 9(7): p. 565-571.

[23] Massaroti, P., et al., Development and validation of a selective and robust LC-MS/MS method for quantifying amlodipine in human plasma. Analytical and bioanalytical chemistry, 2005. 382(4): p. 1049-1054.

[24] Shimooka, K., Y. Sawada, and H. Tatematsu, Analysis of amlodipine in serum by a sensitive high-performance liquid chromatographic method with amperometric detection. Journal of Pharmaceutical and Biomedical Analysis, 1989. 7(11): p. 1267-1272.

[25] Chitlange, S., K. Bagri, and D. Sakarkar, Stability indicating RP-HPLC method for simultaneous estimation of valsartan and amlodipine in capsule formulation. Asian J. Res. Chem, 2008. 1: p. 15-18.

[26] El-Gizawy, S.M., et al., Development and Validation of HPLC Method for Simultaneous Determination of Amlodipine, Valsartan, Hydrochlorothiazide in Dosage Form and Spiked Human Plasma. American Journal of Analytical Chemistry, 2012. 3(6): p. 422-430.

[27] Josefsson, M., A.L. Zackrisson, and B. Norlander, Sensitive highperformance liquid chromatographic analysis of amlodipine in human plasma with amperometric detection and a single-step solid-phase sample preparation. Journal of Chromatography B: Biomedical Sciences and Applications, 1995. 672(2): p. 310-313.

[28] Naidu, K.R., U.N. Kale, and M.S. Shingare, Stability indicating RPHPLC method for simultaneous determination of amlodipine and benazepril hydrochloride from their combination drug product. Journal of pharmaceutical and biomedical analysis, 2005. 39(1-2): p. 147-155.

[29] Dongre, V.G., et al., Simultaneous determination of metoprolol succinate and amlodipine besylate in pharmaceutical dosage form by HPLC. Journal of pharmaceutical and biomedical analysis, 2008. 46(3): p. 583-586.

[30] Ma, Y., et al., Determination and pharmacokinetic study of amlodipine in human plasma by ultra performance liquid chromatography-electrospray ionization mass spectrometry. Journal of pharmaceutical and biomedical analysis, 2007. 43(4): p. 1540-1545.

[31] Kasawar, G.B. and M.N. Farooqui, Simultaneous determination of amlodipine besylate and benazepril hydrochloride in pharmaceutical dosage form by LC. Analytical sciences: the international journal of the Japan Society for Analytical Chemistry, 2009. 25(12): p. 1495.

[32] Barman, R.K., et al., Simultaneous high-performance liquid chromatographic determination of atenolol and amlodipine in pharmaceutical-dosage form. Pak. J. Pharm. Sci, 2007. 20(4): p. 274-279.

[33] $\mathrm{Yu}, \mathrm{Q}$., et al., HPLC-MS-MS for the simultaneous determination of atorvastatin and amlodipine in plasma of hypertensive patients. Chromatographia, 2011. 73(3-4): p. 257-262.

[34] Nirogi, R.V.S., et al., Sensitive and rapid liquid chromatography/tandem mass spectrometry assay for the quantification of amlodipine in human plasma. Biomedical Chromatography, 2006. 20(9): p. 833-842

[35] Bhatt, J., et al., A rapid and sensitive liquid chromatography-tandem mass spectrometry (LC-MS/MS) method for the estimation of amlodipine in human plasma. Biomedical Chromatography, 2007. 21(2): p. 169-175.

[36] Karra, V., et al., Simultaneous determination of losartan, losartan acid and amlodipine in human plasma by LC-MS/MS and its application to a human pharmacokinetic study. Pharmaceutical Methods, 2012. 3(1): p. 18. [37] Feng, Y., et al., Analysis of amlodipine in human plasma by liquid chromatography-mass spectrometry. Journal of chromatographic science, 2002. 40(1): p. 49-53. 